\title{
XV Encuentro Nacional de Óptica - VI Conferencia Andina y del Caribe en Óptica y sus Aplicaciones
}

Promovido por la actual Sociedad Red Colombiana de Óptica, el Encuentro Nacional de Óptica (ENO) ha sido una actividad periódica desde su primera versión realizada en el año 1987 en la ciudad de Medellín. En el año 2006, la Red Colombiana de Óptica amplió la cobertura de promoción, fomento y fortalecimiento de la óptica y sus aplicaciones al invitar grupos de investigación de la región Andina (Chile, Perú, Bolivia y Venezuela) y del Caribe (Panamá, Cuba, Costa Rica, Honduras, El Salvador, Guatemala, Jamaica y República Dominicana) realizando, en el marco del X ENO, la Primera Conferencia Andina y del Caribe en Óptica y sus Aplicaciones (CANCOA).

Desde esa época, y cada dos años, la comunidad de científicos de Colombia y Latinoamérica, cuyas investigaciones se enfocan en cualquier campo de la óptica y sus aplicaciones, se reúnen en el Encuentro Nacional de Óptica y Conferencia Andina y del Caribe en Óptica y sus Aplicaciones (ENO-CANCOA) para compartir experiencias, crear nuevas oportunidades de investigación conjunta y fortalecer viejas relaciones personales y científicas.

Para el año 2017, la Universidad Industrial de Santander asumió la noble y delicada tarea de realizar el XV ENO - VI CANCOA. En el marco de las actividades de conmemoración de los 50 años de funcionamiento de la Escuela de Física de la Universidad Industrial de Santander, se realizó del 20 al 24 de noviembre de 2017 el XV ENO - VI CANCOA en las instalaciones del Hotel Bucarica: sede empresarial, cultural y administrativa de la Universidad Industrial de Santander. El comité organizador definió dos objetivos centrales para el evento. Por un lado, el evento desarrolló estrategias y actividades para promover, fortalecer y fomentar la investigación en óptica y sus aplicaciones buscando un encuentro cómodo, amplio en tiempo y agradable de sus actores: investigadores consolidados, investigadores en formación y empresarios. Por otro lado, basados en el lema: "Identificando actores y compartiendo experiencias construimos futuro", los directores de grupos de investigación colombianos en óptica y sus aplicaciones realizaron ponencias plenarias mostrando las temáticas de investigación más fuertes con resultados destacados a nivel nacional e internacional, cumpliéndose con el objetivo de obtener un mapa actualizado de quien hace qué y dónde en óptica.

Resultado de las actividades realizadas, se recibieron numerosos artículos en extenso de alta calidad científica, que fueron evaluados y clasificados por el comité científico, conformado por destacados investigadores internacionales y nacionales. Como resultado de este delicado proceso de selección, y después de surtir el proceso de evaluación por pares, se presenta en el número 163 de la prestigiosa revista de la Academia Colombiana de Ciencias Exactas, Físicas y Naturales los artículos seleccionados por el comité científico del evento. El comité organizador del XV ENO - VI CANCOA agradece enormemente la colaboración de la doctora Elizabeth Castañeda, editora de la revista y del doctor Román Castañeda, editor asociado de los artículos enviados, por la grandiosa labor de publicar los artículos seleccionados y facilitar la labor de organizar un evento científico.

Jaime Enrique Meneses Fonseca

Presidente XV ENO - VI CANCOA 